\title{
Das Verhalten der echten Nucleinsäure zu Farbstoffen.
}

\author{
II. Mitteilung.
}

Von

R. Feulgen.

(Aus dem physiologischen Institut der Universität Berlin.)

(Der Redaktion zugegangen am 12. März 1913.)

Die Versuche über das Verhalten der echten Nucleinsäure zu Farbstoffen wurden von mir fortgesetzt und ich bin nunmehr in der Lage, meinen früher ${ }^{1}$ ) gegebenen Bericht in mancher Beziehung zu erweitern.

Von etwa 100 der verschiedensten Farbstoffe, die mir Herr Geheimrat Rubner in liebenswürdiger Weise zur Verfügung gestellt hatte, wählte ich 20 der bekanntesten und in biologischer Hinsicht wichtigsten aus und prüfte zunächst im Reagenzglase, ob sie mit einer wässerigen Lösung von nucleinsaurem Natrium Niederschläge gaben. Es stellte sich dabei heraus, daß mit sauren Farbstoffen überhaupt keine Fällungen, mit basischen dagegen fast stets Niederschläge sich erzielen ließen. Eine Ausnahme machte nur das Viktoriablau B (Elberf. Farb.), welches keine Fällung gab. Ich möchte gleich vorweg bemerken, dảß es bei all diesen Versuchen unumgänglich notwendig ist, die Lösung des nucleinsauren Natriums in die des Farbstoffes hineinzugießen; verfährt man dagegen umgekehrt, so erbält man auch mit basischen Farbstoffen häufig gar keine, meistens aber unvollständige Niederschläge, die sich zudem nicht filtrieren lassen. Dies kommt offenbar daher, daß das im Überschuß vorhandene nucleinsaure Natrium auf das entstehende Farbnucleinat als Stützkolloid wirkt und dies in kolloidaler Lösung hält, eine Eigenschạft, die sich bei der Nucleinsäure oft beobachten läßt. So ist es z. B. ganz unmöglich, etwa

1) Diese Zeitschrift, Bd. 80, S 73.

Hr,ppe-Seyler's Zeitschrift r. physiol. Chemie. LXXXIV. 
gefärbte Lösungen von nucleinsaurem Natrium mit Tierkohle zu entfärben, weil es mit keinen Mitteln gelingt, die Kohle wieder vollständig aus der Lösung zu entfernen.

Für eine genauere chemische Untersuchung der Niederschläge kam es nun vor allen Dingen darauf an, solche Farbstoffe zu wählen, die einerseits auch als Base möglichst beständig waren, anderseits aber sich in vollkommen reinem Zustande darstellen ließen; sie mußten also in erster Linie gut krystallisierende Salze bilden. Am meisten geeignet erschienen mir aus diesen Gründen das Malachitgrün und das Krystallviolett. Die Reindarstellung dieser Körper geschieht allgemein, indem man zunächst gut krystallisierende Salze derselben, sodann noch einmal die mittels Alkali abgeschiedenen Basen aus geeigneten Lösungsmitteln umkrystallisiert, und diese Basen dann in die verlangten Salze überführt. Als Säure wählte ich die Chlorwasserstoffsäure, aus dem Grunde, weil sie sich leicht

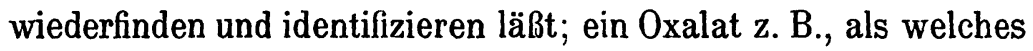
das Malachitgrün in den Handel kommt, war für meine Zwecke unbrauchbar, schon wegen der organischen Natur der Oxalsäure.

Das benutzte nucleinsaure Natrium war nach dem $\mathrm{Neu}$ mannschen Verfahren aus Kalbsthymus dargestellt. Es wies alle Zeichen der Reinheit auf. Die Analyse des Präparates gab folgende Werte :

1. $\mathrm{N}=11,86 \%(\mathrm{Kjeldahl})$,

2. $\mathrm{P}=6,87 \%$ (Neumann).

$\mathrm{N}: \mathrm{P}=1,72$; berechnet 1,70 für $\mathrm{C}_{43} \mathrm{H}_{57} \mathrm{~N}_{15} \mathrm{P}_{4} \mathrm{O}_{34} \mathrm{Na}_{4}$ $+9 \mathrm{H}_{2} \mathrm{O}^{1}$ )

1. Das nucleinsaure Malachitgrünleukohydrat.

(Nucleinsaures Tetramethyldiamidotriphenylcarbinol.)

Das Handelsprodukt des Málachitgrüns (Oxalat, Kahlb a u m) wurde zweimal aus Wasser umkrystallisiert, sodann die Base ${ }^{2}$ ) mittels Natronlauge abgeschieden und diese noch zweimal aus Benzol umkrystallisiert.

1) H. Steudel, Diese Zeitschrift, Bd. 77, S. 497.

2) 0. Fischer, A. 206, S. 132. 
Das Verhalten der echten Nucleinsäure zu Farbstoffen. II. 311

Fp. $132^{\circ}$ (verlangt $\left.132^{\circ}\right) \cdot{ }^{1}$ ) Aus der Base wurde durch Auflösen in der berechneten Menge verdünnter Salzsäure das Chlorid dargestellt und durch Eindampfen bis zur Trockne gewonnen. Das Chlorid krystallisiert nicht. Die Darstellung des Farbnucleinates geschieht nach der in der ersten Mitteilung angegebenen Methode. Es wurde wiederum großes Gewicht auf ein sorgfältiges Auswaschen des Niederschlages gelegt, um eventuell adsorbierten Farbstoff, sowie nucleinsaures Natrium zu entfernen. Da der Niederschlag sich nicht filtrieren ließ, mußte er durch Zentrifugieren gewonnen und ausgewaschen werden. Die Substanz quoll und löste sich dabei, sodaß die Ausbeuten ziemlich mangelhaft waren. Es gelang auch bei diesem zweiten Präparate, den Körper vollständig chlorfrei zu bekommen.

Angenommen, in dem Farbsalze wäre die gleiche Menge Konstitutionswasser enthalten, wie sie H. Steudel²) für das Natriumsalz angegeben hat (9 Mol.), so ergeben sich für das vierbasische Salz $\mathrm{C}_{43} \mathrm{H}_{57} \mathrm{~N}_{15} \mathrm{P}_{4} \mathrm{O}_{34} \cdot 4\left(\mathrm{C}_{23} \mathrm{H}_{25} \mathrm{~N}_{2}\right)+9 \mathrm{H}_{2} \mathrm{O}$ folgende Zahlen:

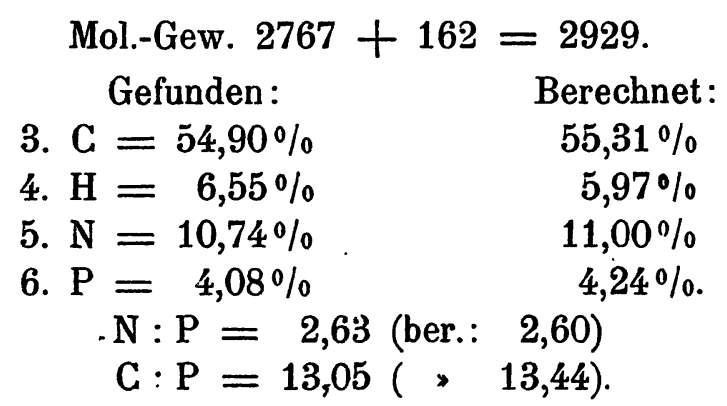

Man beobachtet in dieser Zusammenstellung eine sehr gute Übereinstimmung nicht nur der Quotienten $\mathrm{N}: \mathrm{P}$ und $\mathrm{C}: \mathrm{P}$, sondern auch der absoluten Prozentzahlen mit den berechneten Werten. Daß auch die absoluten Prozentzahlen so gut stimmen, ist natürlich Zufall, weil diese abhängig sind vom Wassergehalt. Da dieser in der Formel nur hypothetisch ist und sich obendrein beim Trocknen in unbekannter Weise ändern kann, so

2) Es wird bei dieser Base Dimorphie mit niedrigerem Fp. beobachtet. v. Villiger u. E. Kopets chki, Ber. d. D. Ch. G., Bd. 45, S. 2910.

2) H. Steudel, Diese Zeitschrift, Bd. 77, S. 497. 
wurde bei diesen wie bei sämtlichen anderen Präparaten auf ein Trocknen bis zur Gewichtskonstanz bei einer bestimmten Temperatur überhaupt verzichtet; maßgebend sind also nur die Quotienten $\mathrm{N}: \mathrm{P}$ und $\mathrm{C}: \mathrm{P}$.

Es wurde sodann das Verhalten dieses Farbnucleinates zu Lösungsmitteln untersucht. Von zahlreichen Solventien übten nur die Alkohole einen Einfluß auf den Körper aus. Übergießt man denselben mit Äthylalkohol, so löst sich immer nur ein Teil zu einer tiefgrünen Flüssigkeit auf, während der Rückstand allmählich immer weniger Farbstoff abgibt und sich vermutlich bis zum farblosen Ablaufen des Alkohols auswaschen läßt. In meiner ersten Mitteilung sprach ich bereits die Vermutung aus, daß sich mit dem Alkohol ein Äther der Farbbase gebildet haben könnte. Das Malachitgrünleukohydrat reagiert nämlich sehr leicht mit Alkoholen, ${ }^{1}$ ) indem das Carbinol desselben mit dem betreffenden Alkohol unter Wasserabspaltung regelrechte gemischte Äther bildet nach der Gleichung:

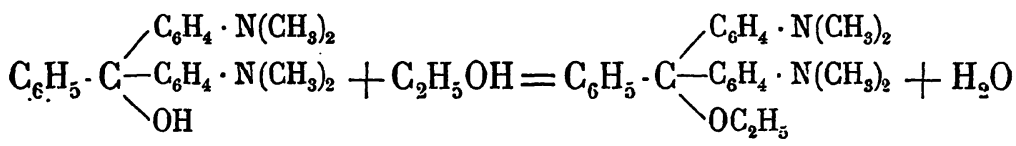

-Diese Reaktion tritt merkwürdigerweise bei der freien Base schon durch bloßes Lösen derselben in Alkohol ein, fast quantitativ vollzieht sie sich aber beim mehrmaligen Umkrystallisieren der Base aus Alkohol oder durch Erhitzen im Rohre. Solche Äther sind vom Methyl-, Äthyl- und Benzylalkohol beschrieben worden.

Um die Substanz auf dieses Verhalten hin zu prüfen, wurde sie mit absolutem Alkohol zu einem dünnen Brei angerührt, zuerst einige Stunden in der Schüttelmaschine geschüttelt, sodann auf dem Wasserbade am Rückflußkühler gekocht und endlich das so vorbehandelte Material der Alkoholextraktion im Soxhlet unterworfen.

Unterläßt man die erwähnte Vorbehandlung, so backt die Aufschwemmung unter dem Einfluß des sich zunächst teigig lösenden Körpers fest zusammen und verhindert so das Eindringen des Lösungsmittels. Die vereinigten Extrakte wurden

i) 0. Fischer, A. 206, S. 132. 
Das Verbalten der echten Nucleinsäure zu Farbstoffen. II. 313

nun eingeengt und einige Tage unter häufigem Reiben stehen gelassen. Es bildete sich am Boden des Gefäßes ein Überzug von schönen grünen Krystallen, die abgesaugt, getrocknet und analysiert wurden. Die trockenen Krystalle erlitten nach Erhitzen auf $100^{\circ}$ keinen Gewichtsverlust.

\section{Gefunden:}

7. $\mathrm{C}=79,95 \%$

8. $\mathrm{H}=8,27 \%$

Fp. $163^{\circ}$ (verl. $162^{\circ}$ ).
Ber. f. d. Äther :

$$
80,15 \%
$$$$
8,08 \% \text {. }
$$

Es handelt sich also in der Tat um eine Ätherbildung. Es ergibt sich nun eine weitere Frage: Was wird aus der Nucleinsäurekomponente, und bin ich imstande, auf diese Weise alle Farbbase als Äther zu extrahieren? Um diese Fragen zu beantworten, mußte vor allem die Reaktion ganz bis zu Ende durchgeführt werden, d. h. es mußte solange im Soxhlet extrahiert werden, bis der Alkohol farblos ablief. Dies hatte jedoch seine Schwierigkeiten; jedenfalls war nach 8 tägigem Extrahieren der Alkohol immer noch schwach grün gefärbt. Der Prozeß wurde aber jetzt unterbrochen; es zeigte sich, daß der Inhalt der Extraktionshülse etwa um die Hälfte leichter geworden war; dies entspricht auch ungefähr dem Gehalt des Farbnucleinates an Farbbase. Der Rückstand wurde analysiert:

9. $\mathrm{N}=12,75 \%$.

10. $\mathrm{P}=8,55 \%$.

Gef.: Ber. für Farbnucl.: Ber. für Nucleinsäure: $\mathrm{N}: \mathrm{P} 1,49^{\circ} \quad 2,60$ 1,70.

Beim Entfernen der Farbbase aus der Verbindung mußte natürlich, da der Phosphorgehalt auf diese Weise zunahm, der Quotient N : P kleiner werden und von 2,60, dem Quotienten des Farbnucleinates, auf 1,70, dem Quotienten der Nucleinsäure, herabsinken. Er erwies sich aber als noch kleiner wie bei der Nucleinsäure, obgleich doch sicher noch Farbbase, wenn auch in geringer Menge, vorhanden war, der Quotient also eher größer sein müßte als 1,70. Es mußite also stickstoffhaltige Substanz, die nicht der Farbbase zukommt, aus dem Inhalte der Extraktionshülse mit entfernt worden sein. Es lag nahe, den 
Inhalt des Siedekolbens auf Purinbasen zu untersuchen: Der Alkohol wurde abgedampft, der Rückstand mit 5\% iger Schwefelsäure hydrolysiert, die Farbbase mittels Natronlauge ausgefällt, und zu dem Filtrat die üblichen Reagenzien auf Purinbasen gegeben: Es entstand sowohl in saurer Lösung mittels Kupfersulfat und Natriumbisulfit, als auch bei alkalischer Reaktion mittels ammoniakalischer Silberlösung ein dichter Niederschlag von Purinkörpern. Phosphorsäure dagegen war nụr in Spuren nachzuweisen. Nunmehr war der ganze Vorgang klar: Unter Alkoholaufnahme hatte sich die Farbbase von der Nucleinsäure getrennt und den in Alkohol löslichen Äther gebildet. Die freie Nucleinsäure aber hat sich, wie das übrigens vorauszusehen war, bei der dauernd hohen Temperatur sekundär weiter zersetzt. Nimmt man das Carbinol als die bindende Gruppe an, so geschieht die Abspaltung der Farbbase unter dem Bilde einer regelrechten Alkoholyse. (Siehe dazu aber meine Bemerkung auf Seite 321.) Diesem Vorgange entspricht folgende Gleichung, in welcher der Nucleinsäurerest als Rechteck, der Farbbasenrest als Dreieck versinnbildlicht wurde:

$$
\nabla \square+\mathrm{C}_{2} \mathrm{H}_{5} \mathrm{OH}=\Delta \mathrm{OC}_{2} \mathrm{H}_{5}+\mathrm{H} \square
$$

2. Das nucleinsaure Krystallviolettleukohydrat. (Nucleinsaures Hexamethylpararosanilin.)

Die Reindarstellung des Krystallvioletts geschieht in derselben Weise wie die des Malachitgrüns. Fp. der Base gef. $197^{\circ}$ (verl. $195^{\circ}$ ).

Elementaranalyse (Dennstedt).
Gef.:
Ber.:
11. $\mathrm{C}=76,96 \%$
$77,06 \%$
12. $\mathrm{H}=8,01 \%$
$8,03 \%$.

Es zeigte sich aber, daß ein wiederholtes Umkrystallisieren der Base gar nicht nötig ist, daß es vielmehr genügt, das käufliche Chlorid zweimal aus Wasser umzukrystallisieren. Da das Arbeiten mit größeren Mengen konzentrierter Farbstofflösung sehr unangenehm, das Nucleinat des Krystallvioletts aber in mancher Beziehung bemerkenswert ist, so gebe ich die für die 
Krystallisation ausprobierten Mengenverhältnisse an und beschreibe zugleich eine Methode, welche ein Operieren mit den feuchten Krystallen nach Möglichkeit einschränkt.

$200 \mathrm{~g}$ Krystallviolett (Kahlbaum) werden in einem Stehkolben von einem Liter Inhalt mit $400 \mathrm{ccm}$ Wasser übergossen, und zwar füge man gleich die ersten Portionen Wasser unter heftigem Umschwenken zu, weil sonst das Krystallviolett leicht am Glase festbackt und sich dann nur langsam löst. Der Inhalt wird unter häufigem Schütteln auf dem Wasserbade bis zur Lösung der Krystalle erhitzt. Um zu. entscheiden, ob sich alles gelöst hat, wird der Kolben heftig umgeschwenkt und einige Zeit ruhig stehen gelasśen; infolge des sich bildenden Wirbels lagern sich etwa ungelöste Teile genau in der Mitte des Bodens ab und können mit einem Glasstabe leicht abgetastet werden. Man filtriert die Flüssigkeit in einen ganz gleichen Kolben, wobei ein Heißwassertrichter unerläßlich ist, erwärmt noch einmal auf dem Wasserbade, um etwa schon ausgefallene Krystalle wieder in Lösung zu bringen, und läßt ruhig erkalten. Am anderen Tage wird die Krystallhaut mit einem Glasstabe durchstochen, die Mutterlauge vorsichtig abgegossen, und der Kolben zum Abtropfen umgekehrt in einen Ring gehängt. Sodann fügt man noch etwa $200 \mathrm{ccm}$ Wasser hinzu, erwärmt und filtriert diesmal in ein starkwandiges, gut angewärmtes Becherglas. Am andern Tage wird die gebildete Krystallhaut abgetragen und verworfen, die Mutterlauge abgegossen und durch umgekehrtes Hinstellen des Becherglases in einer Schale abtropfen gelassen. Endlich bricht man die fest am Glase haftende Krystallmasse los, preßt auf einem Tonteller ab und trocknet auf demselben an der Luft bei Zimmertemperatur. Die Krystalle haben die Zusammensetzung: ${ }^{1}$ )

$$
\overbrace{\mathrm{Cl}}^{\mathrm{C}_{6} \mathrm{H}_{4} \cdot \mathrm{N}\left(\mathrm{CH}_{3}\right)_{2}} \begin{aligned}
& +8 \mathrm{H}_{2} \mathrm{O}=\mathrm{C}_{25} \mathrm{H}_{30} \mathrm{~N}_{3} \mathrm{Cl}+8 \mathrm{H}_{2} \mathrm{O} \\
& \mathrm{Mol} .-\mathrm{G} .=407,8+8 \mathrm{H}_{2} \mathrm{O}=552 .
\end{aligned}
$$

2) Beilstein, Handbuch der org.' Chemie, II, S. 1088 (1896). 
Wegen des Krystallwassergehaltes vermeide man ein Übertrocknen des Präparates.

Eine Besonderheit bei der Darstellung des nucleinsauren Krystallvioletts ergibt sich insofern, als das Krystallviolett in kaltem Wasser nur zu etwa $1-2 \%$ löslich ist. Den Versuch, die Füllung in der Hitze vorzunehmen, will ich wegen der eigenartigen Konsistenz, mit der das Farbnucleinat ausfiel, beschreiben.

$50 \mathrm{~g}$ Krystallviolett wurden in $700 \mathrm{ccm}$ Wasser in der Hitze gelöst, und in diese Flüssigkeit eine warme Lösung von $38,5 \mathrm{~g}$ nucl. Natrium in $300 \mathrm{ccm}$ Wasser unter Umrühren hineingegossen. Es bildete sich schon während des Eingießens eine dünne Gallerte, die aber zunächst noch in der Flüssigkeit gleichmäßig verteilt war, d. h. denselben Raum wie die Flüssigkeit einnahm. Nach wenigen Augenblicken änderte sich jedoc' der Zustand, -indem die Gallerte anfing, sich unter Ablösung vom Glase zu kontrahieren, sodaß sie nunmehr einen kleineren Raum einnahm als die Mutterlauge; es wurde also während der Kontraktion Flüssigkeit ausgepreßt. Dabei nahm die Fällung einen ganz außerordentlich zähen Charakter an. In einem bestimmten Stadium war es z. B. mit Leichtigkeit möglich, die Substanz zu Fäden von über 4 Meter Länge auszuziehen, und ein Ende wurde nur dadurch herbeigeführt, daß beim Ausziehen das Material, infolge der Abkühlung, hart und brüchig wurde. Mit fortschreitender $\mathrm{Ab}$ kühlung zog sich die Masse immer mehr zusammen, bis sie zum Schluß als harter Klumpen, einem Opiumkuchen nicht unähnlich, am Boden lag, während die darüberstehende Flüssigkeit fast farblos geworden war. Offenbar war aller Farbstoff in den Kuchen hineingegangen. Dieser wurde oberflächlich mit Wasser abgespült und im Vakuumexsikkator bei Zimmertemperatur getrocknet. Das Trocknen ging überraschend schnell von statten: nach etwa 36 Stunden war eine Menge von $124 \mathrm{~g}$ vollständig ausgetrocknet; das Gewicht betrug nun noch $75 \mathrm{~g}$, es waren also noch etwa $40 \%$ Wasser in dem Körper. Die Ursache des schnellen Trocknens war die starke Porosität des Farbkuchens. Deswegen ließ die Substanz sich auch leicht 
Das Verhalten der echten Nucleinsäure zu Farbstoffen. II. 317

zu einem groben Pulver zerdrücken; einem Pulverisieren in der Reibschale setzte sie jedoch wegen ihrer Härte einen großen Widerstand entgegen. Das Pulver hatte eine ausgesprochen grüne Farbe. Die Analyse ergab:

13. $\mathrm{N}=13,15 \%$ (Dumas).

14. $\mathrm{P}=4,03 \%$ (Neumann).

$$
\mathrm{N}: \mathrm{P}=3,26 \text { (ber.: 3,05). }
$$

Zweifellos war das Präparat aber sehr unrein, wie es ja nach der Art der Darstellung nicht weiter verwunderlich ist; es gab noch Chlorreaktion, auch war es - nach der Flammenfärbung zu urteilen - noch stark natriumhaltig.

Viel besser eignet sich die Darstellung aus verdünnter Lösung; in diesem Falle entsteht stets ein schöner Niederschlag, der sich leicht durch Dekantieren mit großen Mengen Wasser auswaschen, aber auch überraschend gut an der Saugpumpe abfiltrieren läßt:

$50 \mathrm{~g}$ Krystallviolett werden in 41 Wasser gelöst. Die Farblösung wird am besten durch eine mechanische Rührvorrichtung in kräftiger Bewegung gehalten, und mittels Tropftrichters in dünnem Strahle langsam eine kalte Lösung von $38,5 \mathrm{~g}$ nucleinsaurem Natrium in 21 Wasser zugegeben. Durch diese Anordnung soll vermieden werden, daß der entstehende Niederschlag länger mit dem nucleinsauren Natrium in Berührung kommt. Der Niederschlag wird durch Dekantieren mit Wasser ausgewaschen und schließlich an der Pumpe abfiltriert. Zwar gelingt es nie, das Waschwasser völlig farblos zu erhalten, und es fragt sich, rührt diese Färbung von Spuren unzersetzten Krystallvioletts her, oder ist das Farbnucleinat selbst in Wasser etwas löslich? Eine Stickstoffbestimmung nach Kjeldahl, die ich in $100 \mathrm{ccm}$ vorzunehmen versuchte, ergab, daß sich durch Titration mittels n/10-Lösungen keine Spur Stickstoff nachweisen lieB, obgleich die Farbbase allein einen Stickstoffgehalt von über $10 \%$ hat. Ein kolorimetrischer Vergleich des Waschwassers mit einer stark verdünnten Krystallviolettlösung zeigte, daß im Waschwasser nur etwa $!_{3000} \%$ Farbstoff enthalten war; der Körper kann demnach praktisch 
als unlöslich gelten. Im feuchten Zustande stellt die Substanz einen feinkörnigen, für Wasser sehr durchlässigen, in bezug auf die Farbe unansehnlichen schwarz-braunen Schlamm dar, der nach vollständigem Trocknen sich zu einem ausgesprochen grünen Pulver zerdrücken läßt. Dem vierbasischen Salze kommt die Formel zu:

$$
\begin{gathered}
\mathrm{C}_{43} \mathrm{H}_{57} \mathrm{~N}_{15} \mathrm{P}_{4} \mathrm{O}_{34} \cdot 4\left(\mathrm{C}_{25} \mathrm{H}_{30} \mathrm{~N}_{3}\right)+9 \mathrm{H}_{2} \mathrm{O} . \\
\text { Mol.-Gew. } 3170 .
\end{gathered}
$$

Die Analyse lieferte folgende Werte:

15. $\mathrm{N}=13,16 \%$.

$\left.\begin{array}{l}\text { 16. } \mathrm{P}=4,46 \% \\ \text { 17. } \mathrm{P}=4,44 \%\end{array}\right\}$ Mittel $4,45 \%$.

$$
\mathrm{N}: \mathrm{P}=2,93 \text { (ber. 3,05). }
$$

Da der Körper sich außerdem als chlorfrei erwies, so haben wir offenbar das reine vierbasische nucleinsaure Hexamethylpararosanilin vor uns. Ausbeute: quantitativ. Das Nucleinat hat keinen Schmelzpunkt.

Es wurde nunmehr das Verhalten dieses Salzes zu Lösungsmitteln geprüft. Übergießt man die Substanz mit Methylalkohol, so erweicht sie und geht nach kurzer Zeit völlig in Lösung, nur einige Flocken blieben ungelöst zurück, sie bestanden wahrscheinlich aus verunreinigendem nucleinsaurem Natrium. Durch leichtes Erwärmen kann man die Lösung sehr beschleunigen. Läßt man die intensiv violett gefärbte Flüssigkeit mehrere Tage an der Luft stehen, so dickt sie zu einer sirupösen und im weiteren Verlaufe zu einer zähen fadenziehenden Masse ein, die bald gänzlich erhärtet und dann in Gestalt von glasartigen Platten vom Glase abspringt. Aus seiner methylalkoholischen Lösung läßt sich der Körper durch Äther, Aceton und Amylalkohol wieder abscheiden. War die Substanz in gewöhnlichem Methylalkohol gelöst (Methylalkohol I, Kahlbaum), so erfolgte die Fällung stets in einer schmierigen, nicht filtrierbaren Form. Aus absolutem Methylalkohol jedoch wird das Nucleinat durch abșoluten Äther in schönen transparenten Flocken abgeschieden, die im Kontraste gegen die rotstichige Mutterlauge tief blau gefärbt erscheinen. Da das Farbnucleinat in Wasser unlöslich ist, so sollte man annehmen, daß es sich aus alkoholischer 
Lösung durch Wasser ausfällen ließe. Dies ist aber nicht der Fall. Selbst wenn man einige Tropfen der Lösung in einen großen Überschuß von Wasser gibt, erhält man keine Fällung; diese tritt aber sofort ein, wenn man dem Wasser ein paar Tropfen einer gesättigten wässerigen Lösung von Krystallviolett zusetzt. Die Fällung wird allerdings, ebenso wie die Fällung aus wasserhaltigem Alkohol, schmierig und ganz unfiltrierbar. Das Chlorid der Farbbase (Krystallviolett) spielt hier offenbar dieselbe Rolle wie das Natriumacetat beim Ausfällen des nucleinsauren Natriums oder wie. Baryumacetat beim Ausfällen vieler hochmolekularer organischer Baryumsalze mit Alköhol. Die Lösung des Körpers in Äthylalkohol macht einige Schwierigkeiten; weil das Farbpulver im Alkohol sofort zusammenbackt, sich am Glase festsetzt und sehr schwer löst. Will man eine äthylalkoholische Lösung herstellen, so muß man die Substanz mit etwa der 10 fachen Menge Sand innig mischen und dann in einem Extraktionsapparate extrahieren. Engt man das Extrakt genügend ein, so läßt sich auch hier die gleiche Fällung mit Äther hervorrufen. Die Niederschläge werden am schönsteń, wenn sowohl die Substanz als auch die Lösungs- und Fällungsmittel absolut trocken sind. Man löst den Körper zweckmäßig zu einer 5\% igen Lösung in absolutem Methylalkohol auf und filtriert gleich in etwa das doppelte Volumen Amylalkohol hinein. Der entstandene schöne violette Niederschlag wird an der Saugpumpe abfiltriert und zuerst mit Amylalkohol, dann mit Äther gewaschen. Er ist in Äther vollkommen unlöslich.

Das nucleinsaure Hexamethylpararosanilin ist das zurzeit einzig bekannte Salz der echten Nucleinsäure, das ein organisches Lösungsmittel hat, und ich hielt eine solche Eigenschaft für besonders wichtig, erstens, weil sie diese eigenartige Reinigung gestattet, und zweitens, weil ich durch die Elementaranalyse des eventuell vorher mit geglühtem Natriumsulfat in alkoholischer Lösung ausgetrockneten Salzes Aufschlüsse über das sogenannte Konstitutionswasser der Nucleinsäure zu erbalten hoffte. Tatsächlich läßt sich auch der so vorbehandelte Körper sehr leicht bei $95^{\circ}$ bis zur.Gewichtskonstanz trocknen; 
erhöht man die Temperatur auf $110^{\circ}$, so findet keine weitere Gewichtsabnahme mehr statt. Die Hoffnungen haben sich aber insofern nicht erfüllt, als das mit Amylalkohol ausgefällte Produkt eine andere Zusammensetzung hat als das ursprünglich in Lösung gebrachte. Auffällig war schon, daß die physikalischen Eigenschaften des ausgefällten Körpers erheblich von denen des ursprünglichen abwichen. Das Präparat war nämlich vorher ausgesprochen grün, hinterher aber schön violett gefärbt; vorher war es sehr hart, nachher ließ es sich mit Leichtigkeit $\mathrm{zu}$ feinstem Pulver zerreiben, das dabei stark elektrisch wurde. Vorher löste es sich glatt in absolutem Methylalkohol, nach der ersten oder zweiten Fällung jedoch nur in wasserhaltigem ( $75 \%$ igem). Läßt man diese zweite Lösung längere Zeit stehen, oder erwärmt man sie, so fällt ein Körper aus, der sich nunmehr als unlöslich auch in verdünntem Alkohol erwies. Die Analyse des einmal umgefällten Salzes ergab:

\section{Präparat.}

18. $\mathrm{N}=12,72 \%$ (Dumas).

19. $\mathrm{N}=12,71 \%$,

20. $\mathrm{P}=5,09 \%$ (Neumann).

21. $\mathrm{P}=4,95 \%$

$$
\mathrm{N}: \mathrm{P}=2, \tilde{5} 7 \text { (ber. } 3,05) \text {. }
$$

II. Präparat, getrennt dargestellt.

22. $\mathrm{N}=12,44 \%$ (Dumas).

23. $P=5,58 \%$ (Neumann).

24. $\mathrm{P}=5,57 \%$

$$
\mathrm{N}: \mathrm{P}=2,23 \text { (ber. 3,05). }
$$

Dieses rapide Ansteigen des Phosphorgehaltes auf Kosten des Stickstoffs erklärt sich daraus, daß während seines Aufenthaltes in der alkoholischen Lösung der Körper einen Teil der Farbbase unter Bildung von sauren Salzen abgegeben hat; ich glaube nicht, daß eine weitgehendere Zersetzung stattgefunden hat; dagegen spricht erstens der tadellos saubere Charakter der Fällung, zweitens der Umstand, daß ich mit 
Das Verhalten der echten Nucleinsäure zu Farbstoffen. II. 321

sorgfältig wasserfreiem Material gearbeitet habe; es fehlt also an dem für eine Hydrolyse unbedingt notwendigem Wasser. Endlich aber ist der Eingriff, nämlich die Behandlung.des Salzes mit Methyl- bezw. Amylalkohol in der Kälte ein so milder, daß sogar die freie Nucleinsäure ihm gewachsen zu sein pflegt. Nach dem Quotienten N : P zu urteilen, muß es sich etwa um das zweibasische Salz handeln:

$\begin{array}{ccc} & \text { gef. } & \text { verl. f. d. zweibasische Salz } \\ \mathrm{N}: \mathrm{P} & 2,57 & 2,37 \\ & 2,23 & \end{array}$

Soll aber die Base sich in absoluten Lösungen - ohne Wasseraufnabme - abspalten können, so kommt man mit dem Bilde einer einfachen doppelten Umsetzung bei der Bildung des Farbnucleinates nicht aus; man muß vielmehr eine doppelte Umsetzung unter Wasseraufnahme annehmen, sodaß das gebildete Salz in Wirklichkeit eine Addition darstellt. Der Prozeß würde sich dann folgendermaßen vorstellen lassen ${ }^{1}$ ):

$$
\begin{aligned}
& \triangle \mathrm{Cl}+\mathrm{Na} \square+\mathrm{H}_{2} \mathrm{O}=\triangle \mathrm{OHH} \underline{\mathrm{H}}+\mathrm{NaCl} \\
& \triangle \mathrm{OH} \mid \mathrm{H} \underline{\square} \rightarrow \triangle \mathrm{OH}+\mathrm{H} \square
\end{aligned}
$$

Unter diesen Umständen bedarf auch meine Ansicht von der Wirkung des Äthylalkohols auf das Farbnucleinat des Malachitgrüns einer Einschränkung insofern, als hier eine Alkoholyse nicht notwendig angenommen zu werden braucht, unter der Voraussetzung, daß auch hier ein additionelles Salz vorgelegen hat; d. h., eine Abspaltung der Farbbase braucht nicht deswegen stattgefunden zu haben, weil Alkohol eine große Affinität zum Carbinol der Farbbase hat, sondern sie kann deswegen erfolgt sein, weil das additionelle Salz nur sehr locker gebunden ist. In diesem Falle wäre die Ätherbildung, obgleich das hervorstechendste Moment, etwas ganz Sekundäres. Beim Farbnucleinate des Krystallvioletts kann nur dieser letzte Fall in Frage kommen, weil von der Base des Krystallvioletts eine Ätherbildung nicht bekannt ist.

Übrigens wäre noch eine dritte Möglichkeit denkbar; es kann nämlich beim Nucleinate des Krystallvioletts auch aus

1) Die Zeichen wie auf Seite 314 
dem wasserfreien Salze eine Abspaltung der Base stattfinden unter Aufnahme von Alkohol, indem der Alkoholrest mit der Nucleinsäure einen Ester bildet. Dieser Vorgang scheint mir der am wenigsten wahrscheinliche.

Nun zur Frage: Adsorption oder chemische Bindung?

In Anbetracht der Analysen scheint diese Frage vielleicht müßig; um eine Adsorption des Farbchlorids kann es sich in unseren Fällen auch nicht handeln, aus dem Grunde, weil unsere Substanzen chlorfrei waren, wohl aber wird die Frage diskutabel, wenn man die leichte Abgabe der Farbbase berücksichtigt und dabei an folgenden Versuch ${ }^{1}$ ) denkt:

Die Chloride der basischen Anilinfarben sind in wässeriger Lösung stark dissoziiert. Bringt man in diese Lösungen einen energisch adsorbierenden Körper, z. B. Kohle, so wird die Base von der Kohle adsorbiert, während die Flüssigkeit hinterher wegen des Vorhandenseins von feiner Salzsäure sauer reagiert. Würde in unserem Falle auch nur eine Spur der Base in diesem Sinne von dem kolloidalen nucleinsauren Natrium adsorbiert, so hätte das Auftreten von freier Salzsäure sofort ein Ausfallen von freier Nucleinsäure zur Folge. Der entstehende Niederschlag von Nucleinsäure. könnte nun seinerseits wieder Farbbase adsorbieren und das Spiel würde von neuem beginnen, bis alle Farbbase adsorbiert ist, und die Salzsäure aus dem nucleinsauren Natrium alle Nucleinsäure freigemacht hat, bezw. ein Gleichgewichtszustand eingetreten ist. Als Endprodukte würden wir in einem solchen Falle haben: Chlornatrium und freie Nucleinsäure, an der alle wasserunlösliche Farbbase adsorbiert wäre. Aus einem solchen Körper würde man allerdings ebenso wie bei dem Versuche mit der Kohle - mit Leichtigkeit die Farbbase extrahieren können.

Eine derartige Anschauung wäre nun zweifellos irrig. Vor allem aus dem Grunde, weil das Farbnucleinat des Krystallvioletts völlig in Methylalkohol löslich ist: hier haben wir offenbar einen neuen Körper mit ganz anderen physikalischen Eigenschaften vor uns, Eigenschaften, die - wie die Alkohol-

1) H. Freundlich und G. Losew, Zeitschrift f. physik. Chem. Bd. 59, S. 284 (1907). 
löslichkeit - so grundverschieden von denen der freien Nucleinsäure sind, daß sie nur aus einer Salzbildung erklärt werden können. Ferner fehlt die Analogie mit dem später zu besprechenden Nucleinate der Methylenblaubase, aus dem eine Extraktion von Farbbase nicht möglich ist. Endlich aber ist die freie Nucleinsäure nicht nur gegen verdünnte Mineralsäuren, sondern auch schon gegen Wasser sehr empfindlich, und im Falle einer längere Zeit dauernden Suspension in Wasser wäre eine allmähliche Zersetzung unausbleiblich. Nun machte der Niederschlag des nucleinsauren Malachitgrünleukohydrates einen schmierigen Eindruck, und deswegen kam es mir darauf an, zu entscheiden, ob an der Nucleinsäurekomponente irgend welche Zersetzungen vorgegangen waren.

Ich habe mir daher die Frage gestellt: Ist aller Phosphor tatsächlich als Nucleinsäure gebunden? Bejahendenfalls müßte es mir gelingen, sämtlichen Phosphor in Gestalt von nucleinsaurem Natrium zurückzugewinnen. Übergießt man das Farbnucleinat mit verdünnter Natronlauge, so löst es sich leicht unter Entfärbung auf, indem die farblose Base abgespalten wird. Das sich bildende nucleinsaure Natrium kann man dann in der üblichen W'eise mit Alkohol ausfällen, während die Base in dem Alkohol in Löșung geht. Dementsprechend verfuhr ich folgendermaßen :

2,0548 g Farbnucleinat des Malachitgrüns mit einem Phosphorgehalt von $4,08 \%$ wurden in Wasser aufgeschwemmt und mit möglichst wenig verdünnter Natronlauge versetzt. Die anfangs intensiv grün gefärbte Masse entfärbte sich allmählich unter Abscheidung der Base, die aber infolge der Anwesenheit des nucleinsauren Natriums kolloidal in Lösung blieb und der Flüssigkeit ein milchiges Aussehen verlieh. Durch einstündiges Zentrifugieren mit 8000 Touren ließ sich zwar eine geringe Menge grobkörnigeren Materials abscheiden, der größte Teil war aber auch jetzt noch in Suspension. Es wurde daher auf die mechanische Abscheidung der Base verzichtet und die Flüssigkeit gleich mit etwa dem 8 fachen Volumen Alkohol versetzt. Die Lösung wurde jetzt klar, weil die Base sich in dem Alkohol gelöst hatle. Auf Zusatz von etwas Natriumacetat 
trat wiederum eine milchige Trübung auf, offenbar von ausfallendem nucleinsaurem Natrium. Ein einigermaßen flockiger Niederschlag wurde aber erst erhalten, wenn mittels Essigsüure der größte Teil des Alkalis neutralisiert war; denn das nucleinsaure Natrium fällt am besten bei neutraler oder schwach saurer Reaktion aus. Eine alkalische Reaktion war in unserem Falle aber unbedingt notwendig. um die Farbbase sicher frei zu halten, es kam nur darauf an, den Gehalt an überschüssiger Natronlauge auf ein Mindestmaß zu beschränken. Dies ist nun sehr einfach, weil wir in der Farbbase einen vorzüglichen .Indikator haben. Bei neutraler Reaktion verbindet sich nämlich die Farbbase mit dem Alkohol zu einem Äther (s. o.), welcher grün gefärbt ist; ein minimaler Überschuß von freiem Alkali jedoch bringt den Äther zur Verseifung und die nunmehr abgeschiedene Base ist im Alkohol nur noch physikalisch - also farblos - gelöst. Man setzt also nur soviel Alkali zu, bezw. neutralisiert den Überschuß soweit mit Essigsäure, daß die Flüssigkeit eben farblos erscheint. In diesem Falle hat das gebildete nucleinsaure Natrium Neigung, sich abzusetzen, aber nicht schnell, sondern erst im Verlauf von einigen Tagen. Wenn die über dem Bodensatze stehende Flüssigkeit vollkommen klar geworden ist, wird sie vorșichtig abgegossen, der Bodensatz mit Alkohol ausgewaschen, wiederum in Wasser gelöst, und die Fällung mit Alkohol unter Kontrolle der Reaktion wiederholt. Um zum Schluß das nucleinsaure Natrium in eine für die Filtration geeignete grobflockige Form zu bringen, wird eine letzte Fällung in neutraler Reaktion ausgeführt. Der Niederschlag wurde sodann in der üblichen Weise mit Alkohol und Äther gewaschen und getrocknet. Ausbeute an lufttrockenem Material $=1,0973 \mathrm{~g}$. Analyse:

$$
\begin{aligned}
& \text { 25. } \mathrm{N}=12,15 \% \text { (Kjeldahl). } \\
& \text { 26. } \mathrm{P}=7,06 \% \text { (Neumann). } \\
& \mathrm{N}: \mathrm{P}=1,72 \text { (ber. }=1,70) .
\end{aligned}
$$

Wir haben also zweifellos reines nucleinsaures Natrium vor uns. Es zeigte nur eine ganz geringe Spur von Grünfärbung und gelatinierte in $5 \%$ iger Lösung. 
Es fragt sich nun, wieviel Phosphor haben wir aus dem Farbnucleinate wiedergewonnen? Mit den 2,05 $48 \mathrm{~g}$ Farbnocleinat von 4,08\% Phosphorgehalt haben wir 83,84 mg Phosphor in Reaktion gebracht. Wiedergefunden haben wir $1,0973 \mathrm{~g}$ nucleinsaures Natrium mit einem Phosphorgehalte von 7,06\%, also 77,44 mg P. Das macht aber 92,37\% des im Farbnucleinate enthaltenen Phosphors aus; 7,63\% fehlen also. Man kann aber mit größter Sicherheit annehmen, daß dieses Defizit eine Folge unserer Arbeitsmethode ist; denn die quantitative Bestimmung der Nucleinsäure ist stets eine mißliche Sache und immer mit Verlusten bis zu $6 \%$ und darüber gebunden. ${ }^{1}$ ) Dazu kommt noch die in unserem Falle zwar nötige, aber für die Ausfällung des nucleinsauren Natriums ungünstige alkalische Reaktion. In Anbetracht dessen kommt man zu dem Schlusse, daB in dem vorliegenden Präparate aller Phosphor in Form von Nucleinsäure vorhanden ist, daß also von irgend einer Zersetzung nicht die Rede sein kann.

Viel eleganter kann man aus dem nucleinsauren Krystallviolettleukohỵdrat die Nucleinsäure als Alkalisalz wiedergwinnen. Fügt man zu einer Aufschwemmung dieser Substanz einen Überschuß von Natroniauge, so bildet sich zunächst die lösliche echte Ammoniumbase. ${ }^{2}$ ) Erst nach längerem Stehen, oder schneller beim Erwärmen, geht dieser Körper in die vollkommen wasserunlösliche Pseudoammoniumbase (Carbinol) über; die Umwandlung kann man äußerlich daran erkennen, daß der vorher lackfarbene Inhalt des Gefäßes nunmehr deckfarben wird und die charakteristische Farbe der freien Base annimmt. Diese Base läßt sich im Gegensatze zu dem Malachitgrünleukohydrat leicht mittels der Zentrifuge niederschlagen: ist der Prozeß zu Ende geführt, so muß die darüberstehende Flüssigkeit völlig klar und farblos sein, sie reagiert dann auch stets alkalisch. Die letzten Spuren der Base lassen sich dadurch entfernen, daß man die Flüssigkeit mit etwas Talkum schüttelt und filtriert. Zur Gewinnung des nucleinsauren Natriums wird

1) H. Steudel, Diese Zeitschrift, Bd. 73, S. 473.

2) Hantsch, Osswald, Ber. d. D. Ch. G., Bd. 33, S. 300.

Hoppe-Segler's Zeitschrift r. physiol. Chemie. ixxxrv. 
die Lösung mit Essigsäure neutralisiert - wobei meist wieder eine schwache Violettfärbung auftritt - und mit dem doppelten Volumen Alkohol unter Zugabe von etwas Natriumacetat gefällt. Der Niederschlag wird zum völligen Entfärben mit schwach essigsaurem Alkohol ausgewaschen und eventuell noch ein zweites Mal umgefällt. Eine geringe Violettfärbung ist aber ohne jede Bedeutung.

Aus alledem ersieht man, daß wir in dem Krystallviolett ein vorzügliches Fällungsmittel für die Nucleinsäure haben; dies ist um so wichtiger, als wir ein brauchbares Fällungsmittel für die Nucleinsäure gar nicht besitzen, abgesehen vom Alkohol, der aber regelmäßig da versagt, wo noch andere Substanzen zugegen sind, die auf Zusatz des doppelten Volumens Alkohol ausfallen. Die Fällungsmethoden mittels der Schwermetallsalze sind sehr schlecht, weil eine völlige schonende Entfernung der Metalle aus der Nucleinsäure nicht möglich ist: schon die Zersetzung mit Schwefelwasserstoff bietet Schwierigkeiten, und das Freimachen der Nucleinsäure mit verdünnter Salzsäure aus dem Kupfersalz z. B. wird wegen der großen Empfindlichkeit der Nucleinsäure gegen Mineralsäuren besser vermieden. Die Vorteile der Krystallviolettfällung sind: Sauberer, leicht an der Saugpumpe filtrierbarer Niederschlag, eventuelle Reinigung des Farbnucleinates durch Umfällen aus methylalkoholischer Lösung, elegante und schonende Überführung des Niederschlages in ein beliebiges Akalisalz, leichte quantitative Entfernung der in Wasser völlig unlöslichen Farbbase sowohl aus dem Niederschlage als auch aus der Mutterlauge.

Die Schwierigkeit dieser Methode besteht darin, daß man stets die nucleinsäurehaltige Flüssigkeit in die Farbstofflösung gießen muß, daß man also entweder einen berechneten Überschuß von Farbstoff von vornherein haben, oder aber, wo das nicht möglich ist, sich durch Reagenzglasproben von der Anwesenheit eines Farbüberschusses überzeugen muß. Endlich sind die Farbniederschläge in dem intensiv gefärbten Reaktionsgemisch unsichtbar. Immerhin scheint mir diese Methode in geeigneten Fällen manche Vorteile zu besitzen und der Nachprüfung wert zu sein. 
3. Das Nucleinat der Methylenbläubase.

Dieser Körper wurde in derselben Weise erhalten wie die vorigen. Zweifellos handelt es sich auch hier um Salze der. Nucleinsäure. Ausgegangen bin ich von * Methylenblau medicinale purissimum Höchst». Der Farbstoff roch stark nach Dextrin; dieser Geruch schwand jedoch beim Auswaschen des Nucleinates völlig. Die Fällung war eine saubere und lockere und ließ sich sehr gut an der Pumpe über Filtrierpapier filtrieren. Das ablaufende Waschwasser war beinahe farblos. Bemerkenswert ist das Verhalten dieses Körpers gegenüber Lösungsmitteln : Er erwies sich als völlig indifferent gegen alle möglichen Solventien. Bei keinem der Lösungsmittel habe ich ein Abspalten der Base beobachten können. Offenbar ist in diesem Nucleinate die Affinität zwischen Base und Nucleinsäure eine viel intensivere als bei den besprochenen Salzen. Diese Tatsache steht in einem bemerkenswerten Einklang mit der Erfahrung, daß gerade das Methylenblau vorzugsweise die Chromatinsubstanzen des Zellkernes anfärbt, dagegen konnte ich mich überzeugen, daß das Krystallviolett diese Eigenschaft nicht besitzt; es färbt vielmehr in einem mit Alkohol und Äther fixierten Blutpräparate die roten Blutkörperchen stärker an als die weißen, und in diesen den Kern und das Protoplasma gleich intensiv, das Protoplașma häufig sogar noch intensiver. Auch das Malachitgrün ist keine Kernfarbe par excellence. Ich .beabsichtige nunmehr, das Verhalten der Nucleoproteide zu diesen Farbstoffen zu untersuchen, vielleicht ergeben sich da noch interessante Aufschlüsse über die chemischen Vorgänge beim Anfärben des Zellkernes mit basischen Anilinfarben.

\section{Analytische Belege.}

1. $0,3095 \mathrm{~g}$ sättigten $26,2 \mathrm{ccm}$ n/10-Säure $=11,86 \% \mathrm{~N}$ (Kjeldahl).

2. $0,1330, \quad 16,5, \mathrm{n} / 2$-Lauge $=8,87 \% \mathrm{P}$ (Neumann).

3. 0,1953 . lieferten $0,3932 \mathrm{~g} \mathrm{CO}_{2}=54,90 \% \mathrm{C}$ (Liebig).

4. 0,1953 > , $0,1143>\mathrm{H}_{2} \mathrm{O}=6,55 \% \mathrm{H}$,

5. 0,2344 ? $, 22,0 \mathrm{~cm} \mathrm{~N} ; \mathrm{t}=19^{\circ}, \mathrm{b}=754 \mathrm{~mm}, \mathrm{~N}=10,74 \%$.

6. 0,1791 sättigten $13,2, \cdots \mathrm{n} / 2$-Lauge $=4,08 \% \mathrm{P}$ (Neumann).

7. 0,2373 , lieferten $0,6958 \mathrm{~g} \mathrm{CO}_{2}=79,95 \% \mathrm{C}$ (Dennstedt).

8. $0,2373, \quad 0,1754, \mathrm{H}_{2} \mathrm{O}=8,27 \% \mathrm{H}$, 
328 F eulg en, Das Verhalten der echten Nucleinsäure zu Farbstoffen. II.

9. $0,2154 \mathrm{~g}$ sättigten $19,6 \mathrm{ccm} \mathrm{n} / 10-$ Säure $=12,75 \% \mathrm{~N}$ (Kjeldahl).

10. $0,1173,, 18,1, n / 2$-Lauge $=8,55 \% \mathrm{P}$ (Neumann).

11. 0,2110 , lieferten $0,5954 \mathrm{~g} \mathrm{CO}_{2}=76,96 \% \mathrm{C}$ (Dennstedt).

12. $0,2110 \gg \otimes 0,1510 \diamond \mathrm{H}_{2} \mathrm{O}=8,01 \% \mathrm{H}$,

13. 0,3137 . lieferten $35,6 \mathrm{ccm} \mathrm{N} ; t=17^{\circ}, b=757 \mathrm{~mm}=13,15 \% \mathrm{~N}$.

14. $0,2188 \gg$ sättigten $15,9 \gg \mathrm{n} / 2$-Lauge $=4,03 \% \mathrm{P}$ (Neumann).

15. 0,3071 , lieferten 34,4 , $\mathrm{N} ; \mathrm{t}=16^{\circ}, \mathrm{b}=764 \mathrm{~mm}=13,16^{\circ} \% \mathrm{~N}$.

16. 0,2100 , sättigten $16,9, \mathrm{n} / 2$-Lauge $=4,46 \% \mathrm{P}$ (Neumann).

17. 0,1998 , $, 16,0$, $,=4,44 \% \mathrm{P}$,

18. 0,3410 , lieferten $37,0 \gg \mathrm{N} ; \mathrm{t}=19^{\circ}, \mathrm{b}=772 \mathrm{~mm}=12,72 \% \mathrm{~N}$.

19. $0,3286 \gg, 35,6 \times \mathrm{N} ; \mathrm{t}=18^{\circ}, \mathrm{b}=770 \Rightarrow=12,71 \% \mathrm{~N}$.

20. 0,2199 » sättigten $20,2, \mathrm{n} / 2$-Lauge $=5,09 \% \mathrm{P}$ (Neumann).

21. 0,$2095 ; \rightarrow 18,7 \gg,=4,95 \% \mathrm{P}$

22. 0,3278 »lieferten $34,6 \gg \mathrm{N} ; \mathrm{t}=17^{\circ}, \mathrm{b}=770 \mathrm{~mm}=12,44^{\circ} \% \mathrm{~N}$.

23. $0,2183 \rightarrow$ sättigten $22,0 \Rightarrow \mathrm{n} / 2$-Lauge $=5,58 \% \mathrm{P}$ (Neumann).

24. $0,2008 \gg .22,2 \gg,=5,57 \% \mathrm{P} .$.

25. $0,2271>19,7$ > $>$ /10-Säure $=12,15 \% \mathrm{~N}$ (Kjeldahl).

26. $0,1020>\quad 13,0>\mathrm{n} / 2$-Lauge $=7,06 \% \mathrm{P}$ (Neumann). 